\title{
Perceptual fluency and affect without recognition
}

\author{
PUNAM ANAND \\ Columbia University, New York, New York \\ and \\ BRIAN STERNTHAL \\ Northwestern University, Evanston, Illinois
}

\begin{abstract}
A dichotic listening task was used to investigate the affect-without-recognition phenomenon. Subjects performed a distractor task by responding to the information presented in one ear while ignoring the target information presented in the other ear. The subjects' recognition of and affect toward the target information as well as toward foils was measured. The results offer evidence for the affect-without-recognition phenomenon. Furthermore, the data suggest that the subjects' affect toward the stimuli depended primarily on the extent to which the stimuli were perceived as familiar (i.e., subjective familiarity), and this perception was influenced by the ear in which the distractor or the target information was presented. These data are interpreted in terms of current models of recognition memory and hemispheric lateralization.
\end{abstract}

There is substantial evidence that people can form preferences for stimuli in the absence of recognition memory (e.g., Kunst-Wilson \& Zajonc, 1980; Seamon, Brody, \& Kauff, 1983; Seamon, Marsh, \& Brody, 1984; Wilson, 1979). Demonstration of this phenomenon has typically involved showing that affective responses are more positive for previously presented stimuli than for new stimuli, even when the stimuli are not recognized as familiar.

Several explanations have been offered to account for affect without recognition. Zajonc has viewed the phenomenon as evidence that affective responses are sometimes independent of cognitive processing and that affect can precede cognition (e.g., Zajonc, 1980, 1984; Zajonc \& Markus, 1982; Zajonc, Pietromonaco, \& Bargh, 1982). By contrast, Lazarus (1982) contends that cognitive appraisal is a necessary precondition for affective appraisal and that the absence of recognition does not imply the absence of cognition. Rather, the hypothesis is that affective judgments can be based on cognitive activity in the form of perceptual registration occurring prior to recognition (see, e.g., Gordon \& Holyoak, 1983; Lazarus, 1982, 1984; Lazarus \& McCleary, 1951; Seamon et al., 1983; Seamon et al., 1984).

At present, it is problematic whether Zajonc's or Lazarus's view offers a more accurate account of the relationship between affective responses and cognitive appraisal . Progress in resolving this debate seems to be hampered by the absence of a common language to describe affect and cognition. Consider the protagonists' definitions of

Correspondence may be addressed to Punam Anand, Columbia University, Graduate School of Business, Uris Hall 517, New York, NY 10027. affect. Whereas Zajonc (1984) contends that all emotions, including physiological responses such as arousal and preferences, should be labeled affective responses, Lazarus (1984) claims that certain moods, emotions, and physiological reactions should not be considered affective. Yet, because both Zajonc and Lazarus seem to share the belief that like-dislike ratings can be a reliable means of obtaining affective discriminations, these differences in conceptualization do not represent a serious concern.

A more difficult problem emerges because of differences in Zajonc's and Lazarus's concepts of cognition. According to Zajonc (1984), cognitive appraisal implies awareness of the factors on which an appraisal rests. Absence of recognition or awareness is thus considered as evidence for the absence of cognition. On the other hand, Lazarus (1984) defines cognitive activity as any comprehension, "whether in the form of a primitive evaluative perception or a highly differentiated symbolic process" (p. 124). Accordingly, if cognitive appraisal is not deliberate, reflective, or conscious, it is always present in the form of primitive (unconscious) logic.

Thus, it appears that progress in explaining affect without recognition has been stymied by a lack of agreement in defining the notion of cognition; nor has it been apparent how this definitional problem might be resolved. As Zajonc (1984, p. 118) noted in discussing whether affect can occur without recognition, "the question ... cannot be fully resolved unless we have a full understanding of consciousness. Such an understanding is at the moment beyond our reach." Lazarus (1984, p. 126) echoes this sentiment in stating that "at this level of theory, knowledge, and method Zajonc can no more prove that a cognition is not present in any emotion, much less before it occurs, than I can prove it is present." 
Despite the pessimism about progress in explaining the affect-without-recognition phenomenon, it appears that the requisite theory and method may be available. Recent models of recognition judgments offer a promising starting point for explaining the affect-without-recognition phenomenon by providing a means of measuring cognitive activity prior to recognition, and signal-detection analysis provides a means of testing these notions.

\section{Recognition Judgments}

The view emerging from recent work concerning recognition judgments is that two processes may be invoked when people make judgments of prior occurrence (Atkinson \& Juola, 1973; Mandler, 1980). One process is thought to involve an assessment of how familiar a stimulus appears to be. This assessment of familiarity is believed to depend on a determination of perceptual fluency, or the relative ease with which people can perceive a stimulus (Jacoby \& Dallas, 1981; Jacoby \& Witherspoon, 1982; Johnston, Dark, \& Jacoby, 1985). There is also the notion that recognition judgments can be based on a second, more deliberate process that involves a directed search of memory to determine whether or not a stimulus item was originally presented (Atkinson \& Juola, 1973; Johnston et al., 1985; Mandler, 1980). Here, accurate recognition depends on a successful search that involves the generation of contextual or episodic information related to the stimulus item.

Mandler (1980) offers an example from everyday experience that illustrates the role of perceptual fluency and directed search in recognition judgments:

Consider seeing a man on a bus whom you are sure that you have seen before; you "know" him in that sense. Such a recognition is usually followed by a search process asking, in effect, where could I know him from? Who is he? The search process generates likely contexts (Do I know him from work, is he a movie star, a TV commentator, the milkman?). Eventually the search may end with the insight, That's the butcher from the supermarket! (p.252)

In this illustration, the initial recognition of the person as familiar is likely to have been based on perceptual fluency, whereas the recognition of the person as the butcher involves a directed search of memory.

In the present research, we examine the alternative explanations for the affect-without-recognition phenomenon by recasting the debate in terms of models of recognition judgments. Along these lines, it would seem that for both Zajonc and Lazarus the observation of chance recognition implies an unsuccessful directed search. Also, both would seem to agree that when directed search fails, af fective judgments are guided by perceptual fluency. The difference between Zajonc's and Lazarus's positions can be reduced to alternative interpretations of how perceptual fluency influences judgments when directed search fails.

This difference is illustrated by the alternative conceptions of how stimulus repetition affects perceptual fluency and, thereby, judgments. For those who subscribe to Zajonc's view, perceptual fluency is a process that occurs automatically as a function of actual stimulus repetition or objective familiarity (Seamon et al., 1983, 1984). The greater the actual repetition status, the greater the perceptual fluency and the more positive the affective judgments. Importantly, in this conception, perceptual fluency is not considered to be indicative of cognition because it can occur without awareness or deliberation.

A different scenario is thought to be operative by those who favor Lazarus's notion that affect is cognitively based. According to this view, perceptual fluency depends on the perceived or subjective familiarity of a stimulus regardless of its actual repetition status (Johnston et al., 1985). Thus, the greater the subjective familiarity of a stimulus, the greater the perceptual fluency and the more positive the affective response toward it. And because subjective familiarity is conceived of as involving a matching process undertaken to infer whether or not a stimulus was seen before, it is considered to be cognitive.

To assess these alternative perspectives, several empirical requirements must be met. A context is needed in which (1) there is chance recognition of the stimuli, and (2) affective responses can be associated uniquely with either objective or subjective familiarity. Evidence for Zajonc's view would obtain if affective responses were predominantly influenced by the actual repetition status of the stimulus such that the greater the number of exposures, the more positive the judgments, regardless of the perceived familiarity of the stimulus. Evidence favoring Lazarus's view that affect is cognitively based would emerge if an increase in subjective familiarity were accompanied by more positive judgments. In the following discussion, we preview the methodology employed to investigate these alternative views.

\section{Signal Dectection}

In the present research, we adopt the two-stage signaldetection procedure suggested by Johnston et al. (1985) for assessing the role of perceptual fluency and directed search in judgments. First, subjects are presented a series of target stimulus items, then their recognition judgments of these old target items as well as new foil items are assessed. Following this procedure, a fourfold classification of actual exposure status and judged status emerges: hit (old item judged as old), miss (old item judged as new), false alarm (new item judged as old), and correct rejection (new item judged as new).

The role of perceptual fluency in the form of objective and subjective familiarity can be assessed by examining subjects' affective responses for items classified in the four cells on the basis of their recognition judgments. Assuming that liking increases with increases in perceptual fluency (Seamon et al., 1984), evidence that objective familiarity is the dominant factor would emerge if affective responses were more positive for hits than for false alarms and for misses than for correct rejections. By contrast, evidence for the dominance of subjective familiar- 
ity would obtain if affective responses were more positive for hits than for misses and for false alarms than for correct rejections.

Although the role of objective and subjective familiarity in affective judgments can be assessed by testing the predictions outlined above, this goal can be more directly achieved by comparing the magnitude of affective responses to items classified as false alarms and misses on the basis of recognition (see Johnston et al., 1985). This is because false alarms reflect the perceived exposure status of a stimulus, whereas misses reflect the objective exposure status. Evidence that subjective familiarity is a dominant factor influencing affective responses would obtain if affect is more positive for false alarms than for misses. If, however, misses are preferred to false alarms, or there are no differences in affective responses for these two categories, subjective familiarity would not be considered the dominant factor in affective judgments.

In Experiment 1, these predictions were tested in the context of a dichotic listening task. The procedures were designed to be as similar as possible to those used in Wilson's (1979) study because those procedures were successful in demonstrating affect without recognition. Our subjects listened to a distractor presented in one ear while target stimulus information that they were told to ignore was presented in the other ear. In the subsequent test phase, target stimulus information as well as new foil items were presented. The subjects' recognition of these old and new stimuli was used to classify these responses as hits, misses, false alarms, or correct rejections, and subjects' affect toward these items was used to assess whether or not subjective familiarity was operative. Evidence that subjective familiarity was a dominant factor would be obtained if affective responses for false alarms were more positive than those for misses, whereas the absence of this effect would minimize the plausibility of the assertion.

\section{EXPERIMENT 1}

\section{Method}

Subjects. Twenty-four right-handed male and female undergraduate students served as subjects in groups of 6 to 8 persons. Participation partially fulfilled a course requirement. None of the subjects had participated in prior research involving a dichotic listening task.

Stimulus material. Six simple melodies were constructed. Each melody was of approximately $10-\mathrm{sec}$ duration. Pretests indicated that under ordinary listening conditions the melodies were discriminable when judged for composition but not for preferences. Following Wilson's (1979) procedure, three of the melodies were selected at random for presentation during the stimulus phase of the experiment and were designated the target melodies. The target melodies as well as the three remaining foil melodies were later tested for affect and recognition.

Two-track audio tapes were used for the dichotic listening task. On one track, each of the three target melodies was presented five times. Each target melody was played five times before the next melody was played. The distractor stimulus was a prose passage of about 500 words that required 3 min to present. A transcript of the distractor passage was also constructed in which there were three or four typographical errors per line.
Procedure. The subjects were seated in an enclosed cubicle that prevented them from seeing other subjects or the experimenter. Each cubicle was equipped with a set of headphones and a microphone. The subjects were given a booklet that included the experimental instructions, a transcript of the passage, and the dependent measures. The instructions directed the subjects to shadow the passage transmitted via the headphones by reading it aloud and to place a slash through errors in the transcript of the passage typed in the booklet. The subjects were also informed that their performance was being recorded and that the microphone was there for that purpose. The intent of this procedure was to motivate the subjects to perform the shadowing task accurately and completely. In actuality, shadowing performance was not recorded.

The subjects were then given a practice trial in which they put on the headphones and performed the distractor task. This involved shadowing the passage by repeating aloud what they had heard being played in the right ear and slashing errors in a transcript of the passage. No stimulus was presented in the left ear during the practice trial. The subjects were told to ignore any sounds they heard other than the passage during the main study.

The main experiment followed immediately after the practice session. The procedures used in the main experiment were similar to those used successfully by Wilson (1979) to demonstrate affect without recognition. The subjects performed the distractor task, which was transmitted to the right ear. After the subjects had been performing this task for $30 \mathrm{sec}$, the target melodies were presented in the left ear. To facilitate listening to the distractor task information, it was transmitted at a higher volume $(.05 \mathrm{~dB})$ than the target melodies (see Wilson, 1979).

Upon completion of the distractor task, the subjects were told that they would hear six melodies, three of which had been played during the first phase of the study. After the presentation of each melody, the subjects completed the recognition and affect measures. The order of these measures was counterbalanced. Following Wilson (1979), recognition was measured on a 2-point scale labeled "old" and "new," and affect was measured on a 7-point scale with "dislike" and "like" serving as the polar extremes (see Wilson, 1979). Also in accord with Wilson's procedure, the subjects completed a 3-point scale pertaining to their confidence in their recognition judgments ("sure," "half sure," and "guessing").

\section{Results and Discussion}

Manipulation checks. Because the melodies were randomly selected to serve as targets or foils, it was anticipated that no differences in recognition or affect would be found between targets and foils. As a first step in examining this assumption, the subjects' responses to the three target melodies were combined because their recognition and affect scores did not vary $(F \mathrm{~s}<1)$. A similar procedure was followed for the three foils $\left(F_{\mathrm{s}}<1\right)$.

Signal-detection analysis was then employed to determine whether target melodies and foils differed (Green \& Swets, 1966; Healy \& Kubovy, 1978). This entailed computing $d^{\prime}$, the distance in standard deviation units between the mean of the target distribution and the mean of the foil distribution. The larger the value of $d^{\prime}$, the farther apart are the two distributions and the easier it is to discriminate target from foil. Complete overlap between the two distributions is indicated by $d^{\prime}=0$. The subjects' recognition, affect, and confidence indicated that they were insensitive to differences between the targets and the foils (Table 1).

Because interest centers on the affect-without-recognition phenomenon, it was important to determine whether the 
Table 1

Means and Standard Deviations for Affective Responses, and Recognition Accuracy and $d$ 's for Recognition, Confidence, and Affect, Categorized by Experiment and Treatments

\begin{tabular}{|c|c|c|c|c|c|c|}
\hline & \multirow{2}{*}{\multicolumn{2}{|c|}{$\begin{array}{c}\text { Experiment } 1 \\
\text { Right Ear } \\
\text { Attended }\end{array}$}} & \multicolumn{4}{|c|}{ Experiment 2} \\
\hline & & & \multicolumn{2}{|c|}{$\begin{array}{l}\text { Right Ear } \\
\text { Attended }\end{array}$} & \multicolumn{2}{|c|}{$\begin{array}{l}\text { Left Ear } \\
\text { Attended }\end{array}$} \\
\hline & $M$ & $S D$ & $M$ & $S D$ & $M$ & $S D$ \\
\hline Hits & $5.26^{*}$ & 1.12 & 5.26 & 1.09 & 2.45 & 1.17 \\
\hline False Alarms & 4.73 & 1.16 & 4.59 & 1.02 & 2.06 & 1.15 \\
\hline Misses & 3.95 & 1.29 & 3.41 & 1.46 & 2.00 & 1.07 \\
\hline Correct Rejections & 3.41 & 1.09 & 3.22 & 1.21 & 2.01 & 1.32 \\
\hline Recognition Accuracy & \multicolumn{2}{|c|}{.54} & \multicolumn{2}{|c|}{.52} & \multicolumn{2}{|c|}{.34} \\
\hline$d^{\prime}$ (recognition) & \multicolumn{2}{|c|}{.06} & \multicolumn{2}{|c|}{.08} & \multicolumn{2}{|c|}{-.43} \\
\hline$d^{\prime}$ (confidence) & \multicolumn{2}{|c|}{.05} & \multicolumn{2}{|c|}{.12} & \multicolumn{2}{|c|}{.07} \\
\hline$d^{\prime}$ (affect) & \multicolumn{2}{|c|}{.20} & \multicolumn{2}{|c|}{.16} & \multicolumn{2}{|c|}{-.06} \\
\hline$n$ & \multicolumn{2}{|c|}{24} & \multicolumn{2}{|c|}{11} & \multicolumn{2}{|c|}{8} \\
\hline
\end{tabular}

*Higher numbers indicate more positive affective response.

experimental procedures were successful in producing chance recognition. Evidence for chance recognition would be obtained if the probability of accurately recognizing the targets and foils was .50. This was expected because the subjects were told they had been exposed previously to three of the six melodies that were to be presented (see Wilson, 1979). The subjects' accuracy in recognizing the six melodies (.54) indicated that they did not differ from a chance performance $(.50)$ for targets or foils $(t s<1$; see Table 1).

Affect. A two-stage procedure was used to examine the role of perceptual fluency in affective responses. First, the subjects' recognition responses were used to classify each of the six melodies as a hit, correct rejection, miss, or false alarm. Next, the affective responses to the melodies within each category were averaged. This procedure was considered appropriate because affective responses within each category were not significantly different $(F \mathrm{~s}<1)$. The affective responses for melodies categorized as hits and correct rejections were consistent with other studies in the literature (hits $>$ other three categories combined, $p<.05$, and correct rejections $<$ other three categories combined, $p<.05$ ). These outcomes were not considered further because they are not directly relevant to our test for the presence of subjective or objective familiarity.

The extent of subjective and objective familiarity can be inferred by comparing affective responses toward melodies categorized as misses with those that were false alarms (see Johnston et al., 1985). Means and standard deviations for misses and false alarms are shown in Table 1. An analysis of variance (ANOVA) indicated that false alarms induced more positive affect than did misses $[F(1,22)=13.86, p<.001]$, supporting the view that subjective familiarity can be a more dominant factor than objective familiarity in determining preferences for stimuli that are not recognized accurately.

Finally, we examined whether the order in which the recognition and affect measures were administered might have influenced the subjects' responses. It was found that order did not have a significant effect on responses to these measures $(F \mathrm{~s}<1)$.

These findings offer evidence implicating perceptual fluency in the form of subjective familiarity in the judgment of affect. The finding that stimuli incorrectly perceived as familiar were liked more than those incorrectly perceived as unfamiliar is consistent with this view. In Experiment 2, we attempt to extend this analysis by examining the process by which subjective familiarity might operate.

\section{EXPERIMENT 2}

Our observation that the experience of subjective familiarity is a dominant factor in enhancing positive affect leads to the question of what causes such outcomes. In a recent assessment of this issue, Jacoby, Kelley, and Dywan (1989) suggest that remembering without a corresponding veridical representation involves a misattribution process. Applied to our context, this implies that the inference of subjective familiarity occurs because people perceive the stimulus to match a representation in memory. If this analysis is correct, then degrading the stimulus representation in memory should not only undermine objective familiarity but also subjective familiarity. The latter outcome would be consistent with the view that the degraded stimulus representation in memory undermines the matching process subjects use to infer subjective familiarity and render affective judgments.

As a starting point in designing a test of these notions, we noted that in both Wilson's experiment and Experiment 1 , affect without recognition involved presenting the distractor task in the subject's right ear and the target melodies in the subject's left ear. Such procedures were likely to have taken advantage of the widely recognized efficiencies in hemispheric processing. There is substantial evidence that for right-handed people information presented in the left ear is transmitted primarily to the right hemisphere and information presented in the right ear primarily to the left hemisphere via contralateral pathways (Tucker \& Williamson, 1984). Transmission from an ear to the hemisphere on the same side via ipsilateral pathways is impoverished, especially when both ears receive input (Aitken \& Webster, 1972). Moreover, it is thought that the hemispheres differ in how efficiently they process various types of information. The left hemisphere is thought to be more efficient than the right hemisphere in processing linguistic information (Kimura, 1961, 1966), whereas the right hemisphere is believed to have greater efficiency in processing many aspects of music (Gates \& Bradshaw, 1977).

These notions of hemispheric operation are useful in describing the process by which affect without recognition may have occurred in previous research. Presenting a distractor passage in the right ear was likely to have caused its initial registration in the left hemisphere, which is efficient for processing such information. Similarly, presenting the target melody in the left ear caused its ini- 
tial registration in the right hemisphere, which is efficient in processing this type of information. The latter processing apparently provided a sufficient representation of the melodies to enable the matching process whereby subjective judgments were made about whether or not the stimulus had been heard before.

If this scenario is correct, reversing the ears to which the distractor passage and target melodies are sent should alter the outcomes observed. Specifically, presenting the distractor task in the left ear would cause its initial registration in the right hemisphere, which is inefficient in processing such information. Because the processing of the distractor task was required to complete the shadowing exercise, subjects were likely to have transmitted this information via the corpus callosum to the left hemisphere for efficient processing. While the target melodies could be transmitted in a similar manner to their efficient hemisphere, this was unlikely because the instructions to the subjects were to ignore these stimuli. The latter expectation is consistent with Smith, Chu, and Edmonston's (1977) research suggesting that transmission between hemispheres is unlikely to occur for a secondary task. Even if this transmission did occur, there is likely to be substantial information loss in the transmission process (Porter \& Hughes, 1983).

These observations suggest that the initial transmission of distractor and target to inefficient hemispheres would be likely to limit the accessibility of the target melodies. As a consequence of this limited target-melody accessibility, it was expected that perceptual fluency caused by objective familiarity would be minimal and thus affective responses toward misses would be quite negative. The limited target-melody accessibility would also be expected to undermine perceptual fluency caused by subjective familiarity if such fluency were based on a matching between the target and what people thought they had previously heard. Without accessibility of the target, its subjective familiarity would be reduced and affect would therefore be quite negative. Thus, reversing the attended ear should minimize the difference in affective responses between misses and false alarms previously observed.

\footnotetext{
Method

Subjects. The subjects were 19 right-handed undergraduate students who participated in groups ranging in size from 5 to 8 persons. Participation was motivated by a course requirement. None of the subjects who participated in Experiment 1 was included in the present study.

Procedure. The procedure was similar to that used in Experiment 1 . Subjects were randomly assigned to one of two attendedear conditions. For subjects in the right-ear-attended condition, the distractor task was presented in the right ear (left hemisphere) and the target melodies that subjects were told to ignore were presented in the left ear (right hemisphere). For subjects in the left-ear-attended condition, the ears in which the distractor and target information were presented were reversed. As in the previous study, the subjects' recognition of and affect toward the target and foil melodies served as the critical dependent measures. In addition, measures
}

of the subjects' gender, familial handedness, and past musical training were included to assess the possibility that these individual differences might moderate hemispheric specialization for text and music (see Kellar \& Beaver, 1980; Levy \& Reid, 1978).

\section{Results and Discussion}

Manipulation checks. The three target melodies were combined and a group average was computed because the subjects' recognition, affect, and confidence did not differ among the target melodies $(F \mathrm{~s}<1)$. A similar procedure was used for the three foils $(F s<1)$. The mean $d^{\prime}$ s categorized by treatment are shown in Table 1. For the right-ear-attended condition, signal-detection transformations showed that the subjects' recognition, affect, and confidence were insensitive to differences in the target melodies and foils. The subjects' mean accuracy in recognition did not differ from chance (.52) in the right-earattended condition.

Similar analysis for the left-ear-attended condition indicated no differences between the targets and foils $(F s<1)$. Interestingly, the values for $d^{\prime}$ are negative for recognition and affect. This suggests confusion between the target melodies and foils such that subjects may have mistaken the foils for the targets and vice versa. Thus, it appears that the target-melody familiarity perceived in the right-ear-attended condition was affected adversely in the left-ear-attended condition. This view is consistent with the mean recognition accuracy in the two attendedear conditions (Table 1). Whereas recognition accuracy is not different from chance in the right-ear-attended condition, it is below the chance level in the left-ear-attended condition $[t(17)=2.52, p<.05]$. Finally, it was noted that the order of administering the recognition and affect measures, familial handedness, gender, and past musical training did not vary by treatments $(F \mathrm{~s}<1)$.

Affect. The subjects' recognition responses were used to classify the melodies as hits, misses, false alarms, or correct rejections. Affective responses for the melodies in each of these categories were averaged. Because the pattern of hits and correct rejections was similar to the one observed in Experiment 1, analysis was restricted to the affective responses for false alarms and misses. Means and standard deviations for affective responses are shown in Table 1.

An ANOVA indicated the presence of a significant subjective familiarity effect $[F(1,18)=6.59, p<.03]$. False alarms induced more positive affect than did misses. The main effect for attended ear was also significant $[F(1,18)$ $=16.69, p<.001]$. The subjects who received the melodies in the left ear (right hemisphere) were more positive toward them than were those who received them in the right ear (left hemisphere). These effects were qualified by a marginally significant interaction between familiarity and attended ear $[F(1,18)=3.99, p=.06]$. Simple effects tests indicated that affective responses were more positive for false alarms than they were for misses in the 
right-ear-attended condition $[F(1,10)=18.02, p<.01]$, whereas affective responses did not vary in the left-earattended condition $(F<1)$.

The findings reported in Experiment 2 replicate those observed in the previous study. Subjects in the right-earattended condition exhibited the same pattern of affective responses without recognition toward the target and foil melodies as was found in Experiment 1. These outcomes are consistent with the notion that when distractor information is presented in the hemisphere efficient for its processing, as was likely to be the case in the right-earattended condition, perceptual fluency prompted by subjective familiarity is a dominant factor in affective responses. By contrast, when distractor information is sent to the inefficient hemisphere, such as was likely in the left-earattended condition, perceptual fluency related to objective and subjective familiarity is undermined and affect is uniformly negative. This account is consistent with the signal-detection data and the subjects' recognition accuracy and affective responses.

\section{EXPERIMENT 3}

Although the results of our two experiments appear to be explained in terms of subjective familiarity and hemispheric lateralization, the generality of this finding might be questioned. Specifically, it might be argued that the presentation of the target melodies in a massed fashion (e.g., AAA, BBB, CCC) may have biased the outcomes in favor of the subjective familiarity account. Furthermore, it might be contended that had the target presentation been spaced (e.g., $A B C, A B C, A B C$ ), the target melodies might have been better represented in memory, resulting in support for the objective familiarity view. This line of reasoning seems worthy of investigation because it is consistent with the frequent observation that spaced information is better remembered than massed information (see, e.g., Crowder, 1976).

Experiment 3 was designed to check the robustness of the previous findings. This involved using the same procedures as those used in Experiment 2, with the exception that the target presentation was spaced. If the findings of Experiment 2 are robust, the same effects should be found with spaced presentation, that is, affect is more positive toward false alarms than misses in the right-ear-attended condition but not in the left-ear-attended condition.

\section{Method}

Twenty-one undergraduate students who had not participated in the previous studies served as subjects. They shadowed the information presented in one ear and slashed errors in a transcript of this information, while ignoring the target information presented in the other ear. Then they completed the recognition, affect, and individual difference measures. The procedures were the same as those used in Experiment 2, with one exception. Rather than presenting each target melody multiple times before the next one was presented (e.g., AAA, BBB, CCC), the entire sequence of melodies was presented and the pattern was repeated (e.g., ABC, ABC, $\mathrm{ABC}$ ).
Results and Discussion

Manipulation checks. The three target melodies were combined and a group average was computed because subjects' recognition, affect, and confidence did not differ among the target melodies $(F \mathrm{~s}<1)$. A similar procedure was used for the three foils $(F s<1)$. The mean $d^{\prime}$ s categorized by treatment are shown in Table 2 . For both the right- and left-ear-attended conditions, signal-detection transformations showed that the subjects' recognition, affect, and confidence were insensitive to differences in the target melodies and foils. In addition, the subjects' mean accuracy in recognition did not differ from chance in either condition. It was also found that the order of administering the recognition and affect measures, familial handedness, gender, and past musical training did not vary by treatments $(F \mathrm{~s}<1)$.

Affect. The subjects' recognition responses were used to classify the melodies as hits, misses, false alarms, or correct rejections. Affective responses for the melodies in each of these categories were averaged. Because the pattern of hits and correct rejections was similar to the ones observed previously, analysis was restricted to the affective response for false alarms and misses. Means and standard deviations for affective response are shown in Table 2.

An ANOVA indicated the presence of a significant perceived familiarity effect $[F(1,19)=3.87, p<.05]$. False alarms induced more positive affect than did misses. The main effect for attended ear was also significant $[F(1,19)$ $=8.55, p<.01]$. The subjects who received the melodies in the left ear were more positive toward them than were those who received them in the right ear. These effects were qualified by a significant interaction between familiarity and attended ear $[F(1,19)=4.38, p<.05]$. Simple effects tests indicated that affective responses were more positive for false alarms than they were for misses in the right-ear-attended condition $[F(1,10)=5.66$, $p<.04$ ]; affective responses did not vary in the left-earattended condition $(F<1)$.

Table 2

Experiment 3: Means and Standard Deviations for Affective Responses, and Recognition Accuracy and $d$ 's for Recognition, Confidence, and Affect, Categorized by Treatments

\begin{tabular}{|c|c|c|c|c|}
\hline & \multicolumn{2}{|c|}{$\begin{array}{l}\text { Right Ear } \\
\text { Attended }\end{array}$} & \multicolumn{2}{|c|}{$\begin{array}{l}\text { Left Ear } \\
\text { Attended }\end{array}$} \\
\hline & $M$ & $S D$ & $M$ & $S D$ \\
\hline Hits & $5.32^{*}$ & 2.07 & 2.22 & 1.11 \\
\hline False Alarms & 5.15 & 2.63 & 2.11 & 1.51 \\
\hline Misses & 3.40 & 1.59 & 2.30 & 1.74 \\
\hline Correct Rejections & 3.53 & 1.75 & 2.15 & 1.03 \\
\hline Recognition Accuracy & \multicolumn{2}{|c|}{.51} & \multicolumn{2}{|c|}{.50} \\
\hline$d^{\prime}$ (recognition) & \multicolumn{2}{|c|}{.19} & \multicolumn{2}{|c|}{.00} \\
\hline$d^{\prime}$ (confidence) & \multicolumn{2}{|c|}{.32} & \multicolumn{2}{|c|}{.13} \\
\hline$d^{\prime}($ affect $)$ & \multicolumn{2}{|c|}{.03} & \multicolumn{2}{|c|}{.11} \\
\hline$\underline{n}$ & \multicolumn{2}{|c|}{11} & \multicolumn{2}{|c|}{10} \\
\hline
\end{tabular}

*Higher numbers indicate more positive affective responses. 
The results offer evidence of the robustness of the phenomena previously observed. Whether target items are massed or spaced, the result is the same. Affective responses are more positive for false alarms than for misses in the right-ear-attended condition, whereas no differences in affect are found in the left-ear-attended condition.

\section{GENERAL DISCUSSION}

The present research offers evidence for the affectwithout-recognition phenomenon. Affect is more positive when judgments of stimuli recognized at a chance level are based on subjective familiarity rather than on the actual exposure status of the stimulus. Furthermore, this effect is eliminated under conditions in which the memory representation should be degraded. These observations imply that affect without recognition involves a cognitive process in which an effort is made to match stimulus and memory representation as a basis for subjective judgments of familiarity. When such a matching is possible, a feeling of subjective familiarity enhances positive affect toward the stimulus, whereas this effect is absent when degradation of the stimulus in memory limits the opportunity to perform the matching process.

Although the evidence reported offers support for the view that affect without recognition can be cognitively mediated and that this mediation can involve an assessment of perceived or subjective stimulus familiarity, there may be circumstances in which affect is not cognitively mediated. Indeed, research by Jacoby (see Jacoby, Kelley, \& Dywan, 1989) demonstrates that actual exposure status can have a pronounced influence on affective responses in the absence of recognition. Thus, the most reasonable approach is to investigate when affect without recognition is based on objective and subjective familiarity. The present data also do not rule out the explanation for affect without recognition proposed by Seamon and his colleagues (Seamon et al., 1983, 1984). They hypothesized that affect occurs without recognition because the measurement of these responses causes differences in how the stimulus presentation is accessed. Measures requiring affective responses are thought to induce judgments based on subjects' perceptual fluency or familiarity with the stimulus, whereas recognition measures are believed to prompt memory search. Thus, affect without recognition is explained by the development of perceptual fluency with a stimulus that cannot be reconstructed from memory.

Although this theorizing about the formation of affective judgments is generally in accord with our analysis, the data we present runs afoul of Seamon's view in one respect. Seamon et al. (1984) view perceptual fluency as a process that builds up automatically as a function of repeated stimulus exposures or objective familiarity. This theory would suggest that the affective responses for misses would be more positive than the affective responses for false alarms. By contrast, the present study favors the view that perceptual fluency is more a function of sub- jective than of objective familiarity, and this view is supported by the observation of more positive affect toward stimuli categorized as false alarms than toward those classified as misses.

It should also be noted that the present study does not address Seamon's (Seamon et al., 1983) notion that independent processes guide recognition and affective judgments. This is because it is not possible to investigate the processes underlying recognition and to demonstrate chance recognition simultaneously. Thus, at present, it is problematic whether recognition, like affect, can be mediated by perceptual fluency (see Johnston et al., 1985) or whether recognition and affect evoke different processes (see Seamon et al., 1983). Progress in addressing this issue will require procedures to prompt variation in recognjtion rather than the invariance that is fundamental to investigating affect without recognition.

\section{REFERENCES}

AitKen, L. M., \& Webster, W. R. (1972). Medial geniculate body of the cat: Organization and response to tonal stimuli of neurons in ventral division. Joumal of Neurophysiology, 35, 365-380.

AtKINson, R. C., JuolA, J. F. (1973). Factors influencing speed and accuracy of word recognition. In S. Komblum (Ed.), Antention and Performance $I V$ (pp. 583-612). New York: Academic Press.

Crowder, R. G. (1976). Principles of learning and memory. Hillsdale, NJ: Erlbaum.

Gates, A., Bradshaw, J. L. (1977). The role of cerebral hemispheres in music. Brain \& Language, 4, 403-431.

Gordon, P. C., HolyoAK, K. J. (1983). Implicit learning and generalization of the "mere exposure" effect. Joumal of Personality \& Social Psychology, 45, 492-500.

Green, D. M., \& Swets, J. A. (1966). Signal detection theory and psychophysics. New York: Wiley.

HeAly, A. F., \& Kubovy, M. (1978). The effects of payoffs and prior probabilities on indices of performance and cutoff location in recognition memory. Memory \& Cognition, 6, 544-553.

JACOBY, L.L., \& DALLAS, M. (198I). On the relationship between autobiographical memory and perceptual leaming. Joumal of Experimental Psychology: General, 110, 306-340.

Jacoby, L. L., Kelley, C. M., Dywan, J. (1989). Memory attributions. In H. L. Roediger III \& F. M. Craik (Eds.), Varieties of memory and consciousness: Essays in honour of Endel Tulving (pp. 391-422). Hillsdale, NJ: Erlbaum

JACOBY, L. L., \& WITHERSPOON, D. (1982). Remembering without awareness. Canadian Journal of Psychology, 36, 300-324.

Johnston, W. A., DARK, V. J., \& JACOBY, L. L. (1985). Perceptual fluency and recognition judgments. Journal of Experimental Psychology: Learning, Memory, \& Cognition, 11, 3-11.

Kellar, L. A., \& Beaver, T. G. (1980). Hemispheric asymmetries in the perception of musical intervals as a function of musical experience and family handedness background. Brain \& Language, 20, 24-38.

KimURA, D. (1961). Cerebral dominance and the perception of verbal stimuli. Canadian Joumal of Psychology, 15, 166-171.

KumURA, D. (1966). Dual functional asymmetry of the brain in visual perception. Neurophychologia, 4, 275-285.

KUNST-WILSON, W. R., ZAJONC, R. B. (1980). Affective discrimination of stimuli that cannot be recognized. Science, 207, 557-558.

LAZARUS, R. S. (1982). Thoughts on the relations between emotion and cognition. American Psychologist, 37, 1019-1024.

Lazarus, R. S. (1984). On the primacy of cognition. American Psychologist, 39, 124-129.

Lazarus, R. S., MCCleary, R. A. (1951). Autonomic discrimina- 
tion without awareness: A study of subception. Psychological Review, 58, 113-122.

LeVY, J., \& REID, M. (1978). Variations in cerebral organization as a function of handedness, handposture in writing, and sex. Journal of Experimental Psychology: General, 107, 119-144.

MANDLER, G. (1980). Recognizing: The judgment of previous occurrence. Psychological Review, 87, 252-271.

Porter, R. J., Hughes, L. F. (1983). Dichotic listening to CVS: Method, interpretation, and application. In J. B. Hellige (Ed.), Cerebral hemisphere asymmetry: Method, theory, and application (pp. 177-218). New York: Praeger.

Seamon, J. G., Brody, N., KaUfF, D. M. (1983). Affective discrimination of stimuli that are not recognized: Effects of shadowing, masking, and cerebral laterality. Journal of Experimental Psychology: Learning, Memory, \& Cognition, 9, 544-555.

Seamon, J. G., Marsh, R. L., \& Brody, N. (1984). Critical importance of exposure duration for affective discrimination of stimuli that are not recognized. Journal of Experimental Psychology: Learning, Memory, \& Cognition, 10, 465-469.

Smith, M. A., ChU, J., \& Edmonston, W. E. (1977). Cerebral lateralization of haptic perception: Interaction of responses to Braille and music reveals a functional bias. Science, 197, 689-690.
Tucker, D. M., \& Willinmson, P. A. (1984). Asymmetric neural control systems in human self-regulation. Psychological Review, 91, 185-215.

WILSON, W. R. (1979). Feeling more than we can know: Exposure effects without learning. Journal of Personality \& Social Psychology, 37, 811-821.

Zajonc, R. B. (1980). Feeling and thinking: Preferences need no inferences. American Psychologist, 35, 151-175.

Zajonc, R. B. (1984). On the primacy of affect. American Psychologist, 39, 117-123.

Zajonc, R. B., MArkus, H. (1982). Affective and cognitive factors in preferences. Journal of Consumer Research, 9, 123-131.

Zajonc, R. B., Pietromonaco, P., \& Bargh, J. (1982). Independence and interaction of affect and cognition. In M. S. Clark \& S. T. Fiske (Eds.), Affect and cognition: The Seventeenth Annual Carnegie Symposium on Cognition (pp. 211-227). Hillsdale, NJ: Erlbaum.

(Manuscript received June 5, 1989;

revision accepted for publication November 26, 1990.) 